\title{
EDITORIAL
}

\section{Targeting DUBs to degrade oncogenic proteins}

Targeted protein degradation has emerged as a strategy in cancer therapy. Yang et al. discovered that HBX19818, an inhibitor of the deubiquitinase (DUB) USP10, leads to the dual degradation of spleen tyrosine kinase (SYK) and FLT3, resulting in death of AML cells.

British Journal of Cancer (2020) 122:1121-1123; https://doi.org/10.1038/s41416-020-0728-7

\section{MAIN}

After several decades of a stalemate in the treatment of acute myeloid leukaemia (AML), the last 2 years have witnessed the approval of numerous new targeted drugs for patients with AML. In some cases, mutations in the target (e.g. FLT3 [fms-related tyrosine kinase 3] and IDH1/2 [isocitrate dehydrogenase \{NADP+\}]) predict response to the relevant targeted inhibitor. In other cases, such as with the BCL2 inhibitor venetoclax, mutations in the target itself are not a major predictor of response. In either situation, however, a major challenge moving forward will be to circumvent the resistance that will emerge under the selective pressure of these drugs, as well as to expand the armamentarium of targeted agents for patients with AML.

In this issue of the British Journal of Cancer, Yang et al. focus on protein degradation as an approach to targeted therapy. ${ }^{1}$ Specifically, they discover an approach to the degradation of FLT3 and spleen tyrosine kinase (SYK) through the inhibition of USP10 (ubiquitin-specific protease 10), a deubiquitinating enzyme. FLT3 is recurrently mutated in approximately one-third of AML cases through both internal tandem duplications in the juxtamembrane domain and point mutations in the tyrosine kinase domain. In contrast, SYK is more typically activated through integrin and Fc-receptor signalling ${ }^{2}$ with translocations, such as TEL-SYK, a rare event. ${ }^{3}$ SYK is a cytoplasmic tyrosine kinase, best known for its role in B-cell development, but also demonstrated to play an important role in myeloid signalling. Genetic and pharmacological suppression of SYK have been demonstrated to impair cell growth in vitro and in vivo in AML models. ${ }^{4,5}$ Several biomarkers of response to SYK inhibitors in AML have been reported, including high HOXA9 (homeobox A9) and MEIS1 (myeloid ecotropic viral integration site-1) expression and FLT3 mutations. ${ }^{6,7}$ Indeed, SYK has been reported to activate FLT3 through a direct interaction, a finding corroborated in the current study. ${ }^{1,7}$ In support of these preclinical findings, two orally bioavailable SYK inhibitors, entospletinib and TAK-659, have been investigated in clinical trials in patients with $\mathrm{AML}$ alone and in combination with standard chemotherapy. Early responses have been reported, especially in patients with FLT3-mutated AML and high HOXA9/MEIS1 expression, such as MLL (mixed-lineage leukaemia)-rearranged and NPM1C (cytoplasmic nucleophosmin 1) mutant leukaemia. 8,9

Prior studies report that combination therapy targeting SYK and FLT3 with inhibitors selective for each kinase are synergistic in vitro and improve response in vivo compared with each inhibitor alone in FLT3-mutated AML., ${ }^{70}$ An alternative strategy, however, would be to deploy a small molecule kinase inhibitor that simultaneously targets both, such as the compound TAK-659.
Yang et al. ${ }^{1}$ discovered another parsimonious strategy-to degrade both SYK and FLT3 with a single molecule, that of a deubiquitinase (DUB) inhibitor.

The notion of targeted protein degradation as a cancer therapy has gained momentum in recent years. For example, arsenic trioxide and thalidomide derivatives, drugs reported to have clinical activity prior to mechanistic insight, were later demonstrated to work via protein degradation. Arsenic trioxide targets the PML-RARa (promyelocytic leukaemia-retinoic acid receptor a) fusion protein in acute promyelocytic leukaemia (APL) for proteasomal-mediated degradation. ${ }^{11}$ In multiple myeloma, thalidomide derivatives act as "natural glues" by binding to the E3 ligase cereblon (CRBN) and by redirecting its substrate specificity to bind and degrade IKZF1 (IKAROS family zinc finger 1 and IKZF3, essential transcription factors in this disease. $^{12}$ A second method to degrade proteins uses proteolysis-targeting chimeras (PROTACs) (Fig. 1). ${ }^{13}$ Here, a heterobifunctional molecule can be engineered that is comprised of two distinct chemical moieties: a small molecule that can bind to the target protein of interest bridged by a chemical linker to a second small molecule that binds to an E3 ligase protein, such as CRBN or VHL (Von Hippel Lindau). The PROTAC brings the target and the E3 ligase machinery in close proximity, resulting in ubiquitination of the target protein followed by proteasome-mediated degradation. Such approaches hold promise for drugging difficult cancer targets, such as transcription factor fusions.

Yang et al. ${ }^{1}$ focus on a less explored approach to targeted degradation in cancer-the inhibition of DUBs (Fig. 1). There are two main classes of DUBs: cysteine proteases and metalloproteases, with the ubiquitin-specific proteases (USPs) a subfamily of the cysteine proteases. DUBs cleave the peptide or isopeptide bond between ubiquitin and the substrate protein. One DUB inhibitor under preclinical evaluation is HBX19818, which inhibits the activity of USP7 and USP10. ${ }^{14}$ Yang et al. provide compelling evidence that SYK can be degraded by inhibition of USP10 leading to cell death in AML cells driven by activated SYK or mutant FLT3. The same group had shown previously that USP10 is also the major deubiquitinating enzyme for FLT3. ${ }^{15}$ USP10 inhibition thus leads to dual degradation of FLT3 and SYK, which is beneficial; the combined inhibition of FLT3 and SYK by small molecules is more efficacious than inhibition with either an FLT3 or SYK inhibitor alone in AML. 7,10

There are several advantages to a degrader approach as a cancer therapy. ${ }^{13}$ In principal, there is less need for high systemic drug exposures to maintain sufficient target inhibition in vivo, resulting in fewer off-target effects. Second, degradation 


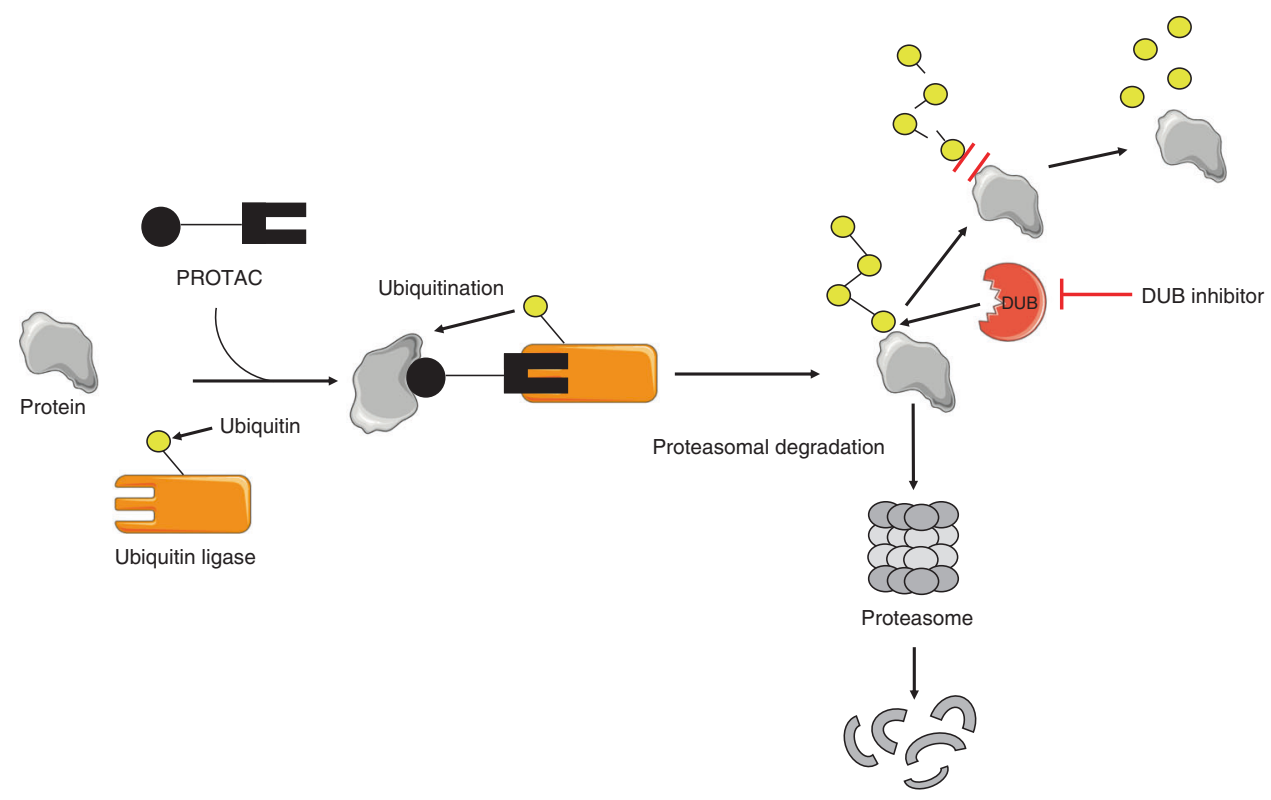

Fig. 1 Targeted protein degradation. Two approaches to targeted protein degradation through activation of proteasomal-mediated degradation are depicted: the use of a PROTAC (proteolysis-targeting chimera) or DUB (deubiquitinase) inhibitor.

strategies may enable the targeting of proteins that have been considered "undruggable", such as scaffolding proteins and transcription factors. Degradation of kinases might also offer additional therapeutic benefit by eliminating both the enzymatic activity and any scaffolding function that the kinase contributes. Moreover, degraders have the ability to counteract the increased target protein expression that frequently accompanies inhibition of protein function. Finally, as the mechanism of resistance to kinases inhibitors is often a new gatekeeper mutation in the kinase itself, degradation eliminates this possibility.

The findings of Yang et al. prompt the more systematic study of the mechanisms by which cancer-associated protein stability is regulated. ${ }^{1}$ With the possibility now of genome-scale CRISPRCas9 screens, one can envision systematic approaches to identify the DUBs/E3 ligases that regulate critical cancer targets. The specific E3 ligases and DUBs involved in the regulation of challenging protein targets might be more easily inhibited by small molecules because they possess enzymatic activity. Moreover, such genome-scale screening approaches can be utilised to anticipate resistance mechanisms to molecules such as HBX19818. Looking to the future, it will be important to test optimised DUB inhibitors in vivo and to dissect the differences in activity of enzymatic inhibitors versus degraders of targets such as SYK and FLT3 alone and in combination.

\section{AUTHOR CONTRIBUTIONS}

A.C and K.S. wrote and approved the final paper.

\section{ADDITIONAL INFORMATION}

Ethics approval and consent to participate N/A

Consent to publish N/A

Data availability N/A

Competing interests K.S. currently has funding from Novartis on topics unrelated to this manuscript and has consulted for Rigel Pharmaceuticals.

Funding information K.S. is funded by National Cancer Institute R35 CA210030-01 and 1P50 CA206963-01. A.C was funded by Deutsche Krebshilfe.
Note This work is published under the standard license to publish agreement. After 12 months the work will become freely available and the license terms will switch to a Creative Commons Attribution 4.0 International (CC BY 4.0).

Publisher's note Springer Nature remains neutral with regard to jurisdictional claims in published maps and institutional affiliations.

Anjali Cremer ${ }^{1,2}$ and Kimberly Stegmaier ${ }^{1}$

${ }^{1}$ Department of Pediatric Oncology, Dana-Farber Cancer Institute and Boston Children's Hospital, Harvard Medical School, Boston, MA 02215, USA and ${ }^{2}$ University Hospital Frankfurt, Department of Hematology/Oncology, Frankfurt/Main, Germany Correspondence: Kimberly Stegmaier (kimberly_stegmaier@dfci.harvard.edu)

\section{REFERENCES}

1. Yang J., Meng C., Weisberg E., Case A., Lamberto I., Magin R. S., et al. Inhibition of the deubiquitinase USP10 induces degradation of SYK. Br J Cancer. https://doi. org/10.1038/s41416-020-0731-z (2020).

2. Miller, P. G., Al-Shahrour, F., Hartwell, K. A., Chu, L. P., Jaras, M., Puram, R. V. et al. In Vivo RNAi screening identifies a leukemia-specific dependence on integrin beta 3 signaling. Cancer Cell 24, 45-58 (2013).

3. Kuno, Y., Abe, A., Emi, N., lida, M., Yokozawa, T., Towatari, M. et al. Constitutive kinase activation of the TEL-Syk fusion gene in myelodysplastic syndrome with $t$ (9;12)(q22;p12). Blood 97, 1050-1055 (2001).

4. Hahn, C. K., Berchuck, J. E., Ross, K. N., Kakoza, R. M., Clauser, K., Schinzel, A. C. et al. Proteomic and genetic approaches identify Syk as an AML target. Cancer Cell 16, 281-294 (2009).

5. Carnevale, J., Ross, L., Puissant, A., Banerji, V., Stone, R. M., DeAngelo, D. J. et al. SYK regulates mTOR signaling in AML. Leukemia 27, 2118-2128 (2013).

6. Mohr, S., Doebele, C., Comoglio, F., Berg, T., Beck, J., Bohnenberger, H. et al. Hoxa9 and Meis1 cooperatively induce addiction to Syk signaling by suppressing miR-146a in acute myeloid leukemia. Cancer Cell 31, 549-562. e11 (2017).

7. Puissant, A., Fenouille, N., Alexe, G., Pikman, Y., Bassil, C. F., Mehta, S. et al. SYK is a critical regulator of FLT3 in acute myeloid leukemia. Cancer Cell 25, 226-242 (2014).

8. Walker, A. R., Byrd, J. C., Bhatnagar, B., Mims, A., Lin, T., Croswell, H. E. et al. Results of a phase 1B/2 Study of Entospletinib (GS-9973) monotherapy and in combination wtih induction chemotherapy in newly diagnosed patients with acute myeloid leukemia. Euro. Hematol. Assoc. Open Access Lib. 214440, S118 (2018). 
9. Kaplan, J., Bixby, D., Morris, J., Frankfurt, O., Altman, J., Wise-Draper, T. et al. a Phase 1b/2 Study of TAK-659, an Investigational Dual SYK and FLT-3 Inhibitor, in Patients (Pts) with Relapsed or Refractory Acute Myelogenous Leukemia (R/R AML). Blood 128, 2834 (2016).

10. Weisberg, E. L., Puissant, A., Stone, R., Sattler, M., Buhrlage, S. J., Yang, J. et al. Characterization of midostaurin as a dual inhibitor of FLT3 and SYK and potentiation of FLT3 inhibition against FLT3-ITD-driven leukemia harboring activated SYK kinase. Oncotarget 8, 52026-52044 (2017).

11. Nasr, R., Guillemin, M. C., Ferhi, O., Soilihi, H., Peres, L., Berthier, C. et al. Eradication of acute promyelocytic leukemia-initiating cells through PML-RARA degradation. Nat. Med. 14, 1333-1342 (2008).
12. Lu, G., Middleton, R. E., Sun, H., Naniong, M., Ott, C. J., Mitsiades, C. S. et al. The myeloma drug lenalidomide promotes the cereblon-dependent destruction of Ikaros proteins. Science 343, 305-309 (2014).

13. Lai, A. C. \& Crews, C. M. Induced protein degradation: an emerging drug discovery paradigm. Nat. Rev. Drug Discov. 16, 101-114 (2017).

14. Reverdy, C., Conrath, S., Lopez, R., Planquette, C., Atmanene, C., Collura, V. et al. Discovery of specific inhibitors of human USP7/HAUSP deubiquitinating enzyme. Chem. Biol. 19, 467-477 (2012).

15. Weisberg, E. L., Schauer, N. J., Yang, J., Lamberto, I., Doherty, L., Bhatt, S. et al. Inhibition of USP10 induces degradation of oncogenic FLT3. Nat. Chem. Biol. 13, 1207-1215 (2017). 